\title{
Thank you from SAMA and the editors
}

\section{The SAMJ reviewers}

SAMA and the editors of SAMJ would like to thank all who participated in the peer review process during 2019. We are grateful for the expertise, insight and thoughtful critiques shared with our authors through the review process, and without which maintaining the high standard of our journal would be impossible.

We apologise if any individual reviewer has inadvertently been excluded, and to those reviewers who were recruited after this issue was published.

\begin{tabular}{|c|c|c|c|}
\hline Amber Abrams & Sam Filby & Johnny Mahlangu & Brian Rayner \\
\hline Pete Acker & Greg Firth & Safia Mahomed & David Rees \\
\hline Miriam Adhikari & Philip Fischer & Aylwyn Mannell & Stephen Reid \\
\hline Ahmad Alli & Rosemary Foster & Relebogile Mapuroma & David Reinders \\
\hline Nazeer Alli & Sasha Frade & Edmore Marinda & Helmuth Reuter \\
\hline Mahmood Ally & Bernd Froessler & Dipuo Masage & Laetitia Rispel \\
\hline Márcio Flávio Araújo & Bryan Fry & Felistas Mashinya, & Rietze Rodeth \\
\hline Affirul Ariffin & Gabriel Gebhardt & Iqbal Master & Chris Rout \\
\hline Yahya Atiya & Theo Gerdener & Angela Mathee & Brian Ruff \\
\hline Chantelle Bagwandeen & Peter Gichangi & Andrew McDonald & Claude Sabeta \\
\hline Robea Ballo & Jeff Gimble & Takafira Mduluza & Shobna Sawry \\
\hline Antoinette Ba-Nhuz & Dominique Goedhals & Ruchika Meel & Janine Scholefield \\
\hline Ahmed Bhigjee & Paul Goldberg & Ushma Mehta & Neshaad Schrueder \\
\hline Prathna Bhola & Lara Nicole Goldstein & Peter Millard, & Muki Shey \\
\hline Shastra Bhoora & Susan Goldstein & Andre Mochan & Joyce Shirinde \\
\hline David Bishop & Ruan Goller & D Modi & Maylene Shung-King \\
\hline Mark Blecher & Perry Gottesfeld & Elizabeth Molyneux & Karen Silwa \\
\hline Marc Blockman & Komalan Govender & Michelle Moorhouse & Melanie Skeen \\
\hline Keith Bolton & Andy Gray & Evelyn Moshkoa & Magda Slabbert \\
\hline Francois Bonnici & Robin Green & Margaret Moss & Martin Smith \\
\hline Martin Botha & Sherika Hanley & Ferdinand Mukumbang & Lisha Sookan \\
\hline Hanneke Brits & Timothy Hardcastle & Saiqa Mullick & Careni Spencer \\
\hline Isabel Brouwer & Di Hawarden & George Muntingh & Dan Stein \\
\hline Stephen Brown & Revyl Hayley & Mageshree Naicker & Victoria Stephen \\
\hline Tawanda Butau & Peter Heering & Nisha Naicker & Chantal Stewart \\
\hline Alex Butchart & Clint Hendrikse & Poovi Naidoo & Elmin Steyn \\
\hline Christo Buys & Sandi Holgate & Saloshini Naidoo & Anne Strode \\
\hline Heloise Buys & Ebrahim Hoosein & Shan Naidoo & Myra Taylor \\
\hline Salome Charalambous & Charlotte Ingram & Gita Naidu & Gerhard Theron \\
\hline Ankia Coetzee & Debra Jackson & Trusha Nana & Jacqueline Thomson \\
\hline Edward Coetzee & Nkosana Jafta & Pradeep Navsaria & Sandie Thomson \\
\hline Marius Coetzee & Stefanus Jansen van Vuuren & Samantha Nicholson & Marianne Tiemensma \\
\hline Cheryl Cohen & Saul Johnson & Nokwasi Nkosi & Andre Troskie \\
\hline Maril Conradie & Josef Jooste & Peter Nourse & Merika Tsitsi \\
\hline Halima Dawood & Ben Jugmohan & Ike Okpechi & Mari van der Vywer \\
\hline Francois de Villiers & Udai Kala & Shahed Omar & Frans Jacob van Wijk \\
\hline Daan den Hollander & Debra Kaminer & Frasia Oosthuizen & Jan van Zyl \\
\hline Lynette Denny & Paul Kapp & Jessica Opie & Richard van Zyl-Smit \\
\hline Wilma Dewitt & Leolin Katsidzira & Muhammad Osman & Per Olav Vandvik \\
\hline Ames Dhai & Mahlatse Kgokolo & Andy Parrish & Sithembiso Velaphi \\
\hline Stewart Dix-Peek & Natasha Khamisa & Nazia Peer & Bonnie Ventor \\
\hline AK Domingo & Aaabida Khan & Olga Perovic & Adriaan Vlok \\
\hline Lissanda du Plessis & Sharon Kling & Velisha Perumal & Klaus von Pressentin \\
\hline Geoffrey Dusheiko & Judy Kluge & Shrikant Peters & Tim Walker \\
\hline Kevin Dzobo & Steven Knight & Diantha Pillay & Nicola Wearne \\
\hline Catherine Egbe & Steve Koch & Melanie Pleaner & Margaret Williams \\
\hline Carina Eksteen & Stephen Korsman & Wolfgang Preiser & Robert Wise \\
\hline Carla Els & Amanda Krause & Rivak Punchoo & Darryl Wood \\
\hline Andreas Engelbrecht & Neo Ledibane & Tanuhsa Ramdin & \\
\hline Harriet Etheridge & Naomi Levitt & Raj Ramesar & \\
\hline June Fabian & Vernon Louw & Leane Ramsoomar & \\
\hline
\end{tabular}




\section{The CME guest editors and authors}

As editor of CME and SAMJ, I would like to thank all the guest editors and authors for their truly outstanding contributions to CME during 2019. This is much appreciated, particularly in the face of increasingly heavy clinical and teaching loads.

\section{Stroke and stroke management in South Africa Guest editors}

A Taylor, Division of Neurosurgery, Department of Surgery, Faculty of Health Sciences, University of Cape Town and Groote Schuur Hospital, Cape Town, South Africa

N A B Ntusi, Division of Cardiology, Department of Medicine, Faculty of Health Sciences, University of Cape Town and Groote Schuur Hospital; Cape Universities Body Imaging Centre, Faculty of Health Sciences, University of Cape Town; and Hatter Institute for Cardiovascular Research in Africa, Department of Medicine, Faculty of Health Sciences, University of Cape Town, South Africa Authors

K Bateman, Division of Neurology, Department of Medicine, Faculty of Health Sciences, University of Cape Town and Groote Schuur Hospital, Cape Town, South Africa

R Harrichandparsad, Department of Neurosurgery, College of Health Sciences, University of KwaZulu-Natal and Inkosi Albert Luthuli Central Hospital, Durban, South Africa

S Whitehead, Life Rehabilitation Unit, Vincent Pallotti Hospital, Cape Town, South Africa

E Baalbergen, Life Rehabilitation Unit, Vincent Pallotti Hospital, Cape Town, South Africa

\section{Health and rehabilitation sciences in a clinical context Guest editor}

N A B Ntusi, Division of Cardiology, Department of Medicine, Faculty of Health Sciences, University of Cape Town and Groote Schuur Hospital; Cape Universities Body Imaging Centre, Faculty of Health Sciences, University of Cape Town; and Hatter Institute for Cardiovascular Research in Africa, Department of Medicine, Faculty of Health Sciences, University of Cape Town, South Africa Authors

N Naidoo, Division of Physiotherapy, Department of Health and Rehabilitation Sciences, Faculty of Health Sciences, University of Cape Town, South Africa

R Barnes, Department of Physiotherapy, School of Allied Health Professions, Faculty of Health Sciences, University of the Free State, Bloemfontein, South Africa

N Mlenzana, Department of Physiotherapy, Faculty of Community and Health Sciences, University of the Western Cape, Cape Town, South Africa

K Mostert, Department of Physiotherapy, Faculty of Health Sciences, University of Pretoria, South Africa

S L Amosun, Division of Physiotherapy, Department of Health and Rehabilitation Sciences, Faculty of Health Sciences, University of Cape Town, South Africa

L Ramma, Division of Communication Sciences and Disorders, Department of Health and Rehabilitation Sciences, Faculty of Health Sciences, University of Cape Town, South Africa

N Schellack, Department of Clinical Pharmacy, School of Pharmacy, Sefako Makgatho Health Sciences University, Pretoria, South Africa

B Heinze, Department of Speech-Language Pathology and Audiology, Faculty of Humanities, University of Pretoria, South Africa

\section{Medical genetics, genomics and the future of medicine} Guest editors

K J Fieggen, Division of Human Genetics, Department of Medicine, Faculty of Health Sciences, Groote Schuur Hospital and University of Cape Town, South Africa

N A B Ntusi, Division of Cardiology, Department of Medicine, Faculty of Health Sciences, University of Cape Town and Groote Schuur Hospital; Cape Universities Body Imaging Centre, Faculty of Health Sciences, University of Cape Town; and Hatter Institute for Cardiovascular Research in Africa, Department of Medicine, Faculty of Health Sciences, University of Cape Town, South Africa Authors

A Krause, Division of Human Genetics, National Health Laboratory Service and School of Pathology, Faculty of Health Sciences, University of the Witwatersrand, Johannesburg, South Africa

K J Fieggen, Division of Human Genetics, Department of Medicine, Faculty of Health Sciences, Groote Schuur Hospital and University of Cape Town, South Africa

L A Lambie, Genetics National Reference Laboratory, Ampath Trust, Pretoria, and Division of Human Genetics, School of Pathology, Faculty of Health Sciences, University of the Witwatersrand, Johannesburg, South Africa

K A Donald, Division of Developmental Paediatrics, Department of Paediatrics and Child Health, Red Cross War Memorial Children's Hospital, Faculty of Health Sciences, University of Cape Town, South Africa

N Laing, Division of Human Genetics, Department of Medicine, Faculty of Health Sciences, University of Cape Town and Groote Schuur Hospital, Cape Town, South Africa

S M Kraus, Division of Cardiology, Department of Medicine, Faculty of Health Sciences, University of Cape Town and Groote Schuur Hospital, Cape Town; and The Cardiovascular Genetics Laboratory, Hatter Institute of Cardiovascular Research in Africa, Department of Medicine, Faculty of Health Sciences, University of Cape Town, South Africa

G Shaboodien, The Cardiovascular Genetics Laboratory, Hatter Institute of Cardiovascular Research in Africa, Department of Medicine, Faculty of Health Sciences, University of Cape Town, South Africa

N A B Ntusi, Division of Cardiology, Department of Medicine, Faculty of Health Sciences, University of Cape Town and Groote Schuur Hospital, Cape Town; The Cardiovascular Genetics Laboratory, Hatter Institute of Cardiovascular Research in Africa, Department of Medicine, Faculty of Health Sciences, University of Cape Town; and Cape Universities Body Imaging Centre, Faculty of Health Sciences, University of Cape Town, South Africa

R S Ramesar, MRC Research Unit for Genomic and Precision Medicine, Division of Human Genetics, Department of Pathology, Institute of Infectious Diseases and Molecular Medicine, Faculty of Health Sciences, University of Cape Town, South Africa

J de Vries, Department of Medicine, Faculty of Health Sciences, University of Cape Town, South Africa

N S Munung, Department of Medicine, Faculty of Health Sciences, University of Cape Town, South Africa 


\section{Diabetes in pregnancy}

Guest editors and authors

S Adam, Department of Obstetrics and Gynaecology, Faculty of Health Sciences, University of Pretoria, South Africa

S Dias, Biomedical Research and Innovation Platform (BRIP), South African Medical Research Council, Cape Town; and Department of Obstetrics and Gynaecology, Faculty of Health Sciences, University of Pretoria, South Africa

P Rheeder, Department of Internal Medicine, Faculty of Health Sciences, University of Pretoria, South Africa

C Pheiffer, Biomedical Research and Innovation Platform (BRIP), South African Medical Research Council, Cape Town; and Division of Medical Physiology, Department of Biomedical Science, Faculty of Medicine and Health Sciences, Stellenbosch University, Cape Town, South Africa

\section{Haematological malignancies Guest editors}

V J Louw, Chair and Head, Division of Clinical Haematology, Department of Medicine, University of Cape Town and Groote Schuur Hospital, Cape Town, South Africa

N A B Ntusi, Division of Cardiology, Department of Medicine, Faculty of Health Sciences, University of Cape Town and Groote Schuur Hospital; Cape Universities Body Imaging Centre, Faculty of Health Sciences, University of Cape Town; and Hatter Institute for Cardiovascular Research in Africa, Department of Medicine, Faculty of Health Sciences, University of Cape Town, South Africa Authors

K Antel, Division of Haematology, Department of Medicine, Faculty of Health Sciences, University of Cape Town, South Africa

E Verburgh, Division of Haematology, Department of Medicine, Faculty of Health Sciences, University of Cape Town, South Africa

B L Houston, Section of Hematology and Medical Oncology, Department of Internal Medicine, Max Rady College of Medicine, Rady Faculty of Health Sciences, University of Manitoba, Winnipeg; and Department of Medical Oncology and Hematology, CancerCare Manitoba, Winnipeg, Canada

E Rimmer, Section of Hematology and Medical Oncology, Department of Internal Medicine, Max Rady College of Medicine,
Rady Faculty of Health Sciences, University of Manitoba, Winnipeg; and Department of Medical Oncology and Hematology, CancerCare Manitoba, Winnipeg, Canada

R Zarychanski, Section of Hematology and Medical Oncology, Department of Internal Medicine, Max Rady College of Medicine, Rady Faculty of Health Sciences, University of Manitoba, Winnipeg; and Department of Medical Oncology and Hematology, CancerCare Manitoba, Winnipeg, Canada

M Seftel, Division of Hematology, Department of Medicine, University of British Columbia, Vancouver, Canada

F Fazel, Division of Clinical Haematology, Department of Internal Medicine, Faculty of Medicine and Health Sciences, Tygerberg Academic Hospital and Stellenbosch University, Cape Town, South Africa

F Bassa, Division of Clinical Haematology, Department of Internal Medicine, Faculty of Medicine and Health Sciences, Tygerberg Academic Hospital and Stellenbosch University, Cape Town, South Africa

P F Wessels, Ampath Laboratories; and Department of Medical Oncology, Faculty of Health Sciences, University of Pretoria, South Africa

C E Menard, Section of Hematology and Medical Oncology, Department of Internal Medicine, Max Rady College of Medicine, Rady Faculty of Health Sciences, University of Manitoba, Winnipeg; and Department of Medical Oncology and Hematology, CancerCare Manitoba, Winnipeg, Canada

A Ponnampalam, Section of Hematology and Medical Oncology, Department of Internal Medicine, Max Rady College of Medicine, Rady Faculty of Health Sciences, University of Manitoba, Winnipeg; and Department of Medical Oncology and Hematology, CancerCare Manitoba, Winnipeg, Canada

\section{Bridget Farham \\ Editor \\ ugqirha@iafrica.com}

S Afr Med J 2019;109(12):895-897. https://doi.org/10.7196/SAMJ.2019.v109i12.14484 